\title{
DIRECT PRODUCTS OF MODULES AND THE PURE SEMISIMPLICITY CONJECTURE
}

\author{
Birge Huisgen-Zimmermann and Frank OKOH \\ Professor Helmut Lenzing anlaesslich seines 60. Geburtstags gewidmet
}

\begin{abstract}
Whenever global decomposition problems involving all modules over a ring are at stake, the 'resistance' of infinite direct products to being decomposed into manageable direct summands plays a crucial role. This phenomenon already surfaced in work of Koethe and Cohen-Kaplansky [14, 5] and in Chase's landmark paper [3], as well as in numerous articles that have appeared since, e.g., in work of Warfield, Griffith, Auslander, Gruson-Jensen, W. Zimmermann, and the first author (see, e.g., $[\mathbf{1 9}, \mathbf{7}, \mathbf{2}, \mathbf{8}, \mathbf{2 0}, \mathbf{1 1}]$ ). While, in the meantime, major headway has been made towards identifying and analyzing the rings whose module categories enjoy the most useful decomposition properties - such as decomposability of all left modules into indecomposable direct summands or, equivalently, into finitely generated components - it is still not clear whether these demands on the base ring are left-right symmetric. The problem is all the more tantalizing as the two-sided requirement is known to coincide with finite representation type $([\mathbf{1}],[\mathbf{1 6}],[\mathbf{6}])$; resolving it thus amounts to locating the missing piece towards a link between finite and infinite dimensional representation theory. We refer to [18] and [12] for more detail on the current status of the problem. The question on which we focus here was raised in its present form by the second author, in pursuance of problems he had jointly tackled with Lenzing [15]. It is also representative of the hurdles that remain in the way of understanding the symmetry issue we sketched. Indeed, a positive answer for all two-sided artinian rings would settle symmetry in the positive.
\end{abstract}

Question. For which rings $\mathrm{R}$ does the following hold: If $\left(M_{n}\right)_{n \in \mathbb{N}}$ is any family of pairwise non-isomorphic finitely generated, indecomposable left $R$ -

The research of the first author was partially supported by a grant from the National Science Foundation.

Typeset by $\mathcal{A M S}_{\mathcal{M}} \mathrm{T}_{\mathrm{EX}}$ 
modules, then the direct product $\prod_{n \in \mathbb{N}} M_{n}$ fails to be a direct sum of finitely generated submodules?

We point out that a negative answer to the symmetry question for global decompositions is expected [17], whence, a fortiori, existence of artinian counterexamples to our product condition appears likely.

The purpose of this note is to exhibit several important instances in which the product condition is satisfied; we hope that our sketch of the base line will trigger further work in this direction. In particular, we will see that all Artin algebras are among the rings satisfying the condition, as well as all commutative noetherian domains of Krull dimension 1. With a modest amount of new input, one can glean these results from the existing literature. While, in complete absence of finiteness conditions for $R$, the outcome is negative just let $R$ be an infinite direct product of local rings $R_{n}$ and choose $M_{n}=R_{n}$ - we conjecture that our product condition is satisfied by all noetherian PI rings. In the noetherian case, decomposability of $\prod_{n \in \mathbb{N}} M_{n}$ into finitely generated submodules of course implies decomposability into indecomposable summands. In view of our example, we will significantly increase the class of rings falling on the positive side by weakening the above product condition, so as to only demand the following: There is no infinite family of pairwise non-isomorphic finitely generated and indecomposable left $R$-modules whose direct product is a direct sum of finitely generated indecomposable components.

We start by making the connection of our question with global decomposition problems more precise. It is well-known that the rings all of whose left modules are direct sums of finitely generated (or indecomposable) submodules are precisely those over which all pure inclusions split $[\mathbf{2 1}, \mathbf{1 1}]$. Their name reflects this behavior: they have been dubbed left pure semisimple rings. In [3], Chase observed that they are necessarily left artinian. As already mentioned, it is known that left and right pure semisimplicity is tantamount to finite representation type, but so far, no examples are available indicating that one-sided pure semisimplicity does not automatically carry over to the other side. In the presence of mild commutativity conditions, the property is in fact known to be left-right symmetric; namely, for Artin algebras [2], and, more generally, for Artin PI rings and rings permitting a (Morita) self-duality $[\mathbf{9}, \mathbf{1 0}]$.

Observation 1. If all two-sided artinian rings satisfy the above product condition, then each left pure semisimple ring has finite representation type.

Proof. According to $[\mathbf{1 3}]$, it suffices to show that all two-sided artinian, left pure semisimple rings are as claimed. So suppose that $R$ is left pure semisimple, artinian on both sides, and satisfies our product condition. Since every 


\section{PRODUCTS AND PURE SEMISIMPLICITY}

left $R$-module and thus, in particular, every direct product of modules is a direct sum of finitely generated components in this situation, we infer that any family of pairwise non-isomorphic finitely generated indecomposable left $R$-modules is finite.

Next we turn to Artin algebras. In this setting, our product property is an immediate consequence of a result due to Auslander [2].

Proposition 2. If $R$ is an Artin algebra and $\left(M_{n}\right)_{n \in \mathbb{N}}$ a family of pairwise non-isomorphic finitely generated, indecomposable $R$-modules, then $\prod_{n \in \mathbb{N}} M_{n}$ is not a direct sum of finitely generated submodules.

Proof. First we observe the following elementary fact: If $R$ is an Artin algebra, and $M=\prod_{n \in \mathbb{N}} M_{n}$ a countable direct product of nonzero left $R$ modules, then $M$ is not countably generated: Clearly, this is true when $R$ is a field, and hence also when $R$ is commutative. Suppose that $R$ is non-commutative, let $C$ be the center of $R$, and $J(C)$ the Jacobson radical of $C$. Since $C / J(C)$ is a finite direct product of fields and $J(C) M_{n}$ is properly contained in $M_{n}$ for each $n$, the $R / J(C)$-module $M / J(C) M=$ $\prod_{n \in \mathbb{N}} M_{n} / J(C) M_{n}$ is not countably generated by the opening remark. Hence, neither is $M$ over $R$. Now suppose $\left(M_{n}\right)_{n \in \mathbb{N}}$ is a family of pairwise nonisomorphic finitely generated indecomposable modules over an Artin algebra $R$. If we had $\prod_{n \in \mathbb{N}} M_{n}=\bigoplus_{i \in I} Q_{i}(\dagger)$ with finitely generated $Q_{i}$, the above fact would force $I$ to be uncountable. On the other hand, each of the $Q_{i}$ would be isomorphic to some $M_{k}$ by Auslander's Cor. 3.2 [2], whence at least one $Q_{j}$ would occur twice in the right-hand side of $(\dagger)$, i.e. $Q_{j} \cong Q_{k} \cong M_{n(j)}$ with $j \neq k$. Now modules with local endomorphism rings can be canceled from direct sum decompositions, which shows that $\prod_{n \in \mathbb{N} \backslash\{n(j)\}} M_{n} \cong \bigoplus_{i \in I \backslash\{j\}} Q_{i}$. But the appearance of $Q_{j}$ in the latter sum contradicts Auslander's result, in view of the fact that the $M_{n}$ are pairwise non-isomorphic.

Finally, we prove the product condition for a substantial class of commutative noetherian rings.

Proposition 3. Suppose that $R$ is a commutative noetherian domain of Krull dimension 1 and $\left(M_{n}\right)_{n \in \mathbb{N}}$ a family of pairwise non-isomorphic finitely generated, indecomposable $R$-modules. Then $\prod_{n \in \mathbb{N}} M_{n}$ is not a direct sum of finitely generated submodules.

Proof. Let $R$ and $\left(M_{n}\right)$ be as in the hypothesis, and assume that $M:=$ $\prod_{n \in \mathbb{N}} M_{n}=\bigoplus_{i \in I} Q_{i}$ with $Q_{i}$ finitely generated. Since, for any nonzero ideal $A$, the factor ring $R / A$ is artinian, the setup clearly implies that $A$ does not annihilate a cofinite subfamily of the family $\left(M_{n}\right)$; for if $A M_{n}=0$ for all but 
finitely many $n \in \mathbb{N}$, say for $n \in N^{\prime}$, one is dealing with a decomposition of the module $M / A M=\prod_{n \in N^{\prime}} M_{n} \oplus \prod_{\mathbb{N} \backslash N^{\prime}} M_{n} / A M_{n}$ over $R / A$ and, after canceling, one obtains a contradiction to the fact that commutative artinian rings satisfy the conjecture.

All $M_{n}$ being noetherian, we can find finitely generated torsionfree submodules $U_{n} \subseteq M_{n}$ such that each of the quotients $M_{n} / U_{n}$ is a torsion module. Moreover, we let $A_{n}$ be the annihilator of $M_{n} / U_{n}$ for $n \in \mathbb{N}$, pick any maximal ideal $B$ of $R$, and let $C_{1}, C_{2}, C_{3}, \ldots$ be an enumeration of all finite products of ideals in the set $\left\{A_{n} \mid n \in \mathbb{N}\right\} \cup\left\{B^{n} \mid n \in \mathbb{N}\right\}$. Then $\bigcap_{n \in \mathbb{N}}\left(C_{n} M_{k}\right)=0$ for all $k \in \mathbb{N}$, and thus $\bigcap_{n \in \mathbb{N}}\left(C_{n} M\right)=0$, while, for each $m \in \mathbb{N}, \bigcap_{n \leq m}\left(C_{n} M_{k}\right) \neq 0$ for infinitely many $k \in \mathbb{N}$ by the initial comment. On the other hand, an extension of Chase's result in [4] (see [12, Lemma 11] for a precise statement and proof of the upgraded version) yields a natural number $L$ such that

$$
\prod_{k \geq L}\left(\bigcap_{n \leq L}\left(C_{n} M_{k}\right)\right) \subseteq \bigoplus_{i \in I^{\prime}} Q_{i}+\bigoplus_{i \in I}\left(\bigcap_{n \in \mathbb{N}}\left(C_{n} Q_{i}\right)\right)
$$

where $I^{\prime}$ is a finite subset of $I$. But the last summand on the right-hand side of this inclusion is zero by construction, whereas the left-hand side is still an infinite product. This forces an infinite product of nonzero modules over the noetherian ring $R$ to be finitely generated, an obvious impossibility.

Concluding Remarks: A slight modification of this argument actually goes through for rings which are module-finite over a central noetherian subdomain of Krull dimension 1. More general results (not just for commutative rings) should be available, via noetherian induction, for classes of noetherian rings $R$ having the following two properties: (i) each proper factor ring of $R$ satisfies the conjecture, and (ii) for every finitely generated left $R$ module $M$ there exists a countable family $\left(C_{n}\right)_{n \in \mathbb{N}}$ of nonzero ideals such that $\bigcap_{n \in \mathbb{N}}\left(C_{n} M\right)=0$.

\section{REFERENCES}

1. M. Auslander, Representation theory of Artin algebras II, Communic. in Algebra 1 (1974), 293-310.

2. L L L L modules over Artin algebras, in Algebra, topology and categories (A. Heller and M. Tierney, eds.), Academic Press, New York, 1976, pp. 1-17.

3. S.U. Chase, Direct products of modules, Trans. Amer. Math. Soc. 97 (1960), 457-473.

4.

5. I.S. Cohen and I. Kaplansky, Rings for which every module is a direct sum of cyclic modules, Math. Zeitschr. 54 (1951), 97-101.

6. K.R. Fuller and I. Reiten, Note on rings of finite representation type and decompositions of modules, Proc. Amer. Math. Soc. 50 (1975), 92-94. 


\section{PRODUCTS AND PURE SEMISIMPLICITY}

7. P.A. Griffith, On the decomposition of modules and generalized left uniserial rings, Math. Ann. 184 (1970), 300-308.

8. L. Gruson and C.U. Jensen, Deux applications de la notion de L-dimension, C. R. Acad. Sci. Paris, Sér. A 282 (1976), 23-24.

9. I. Herzog, Elementary duality for modules, Trans. Amer. Math. Soc. 340 (1993), 37-69.

10. _ A test for finite representation type, J. Pure Appl. Algebra 95 (1994), 151182.

11. B. Huisgen-Zimmermann, Rings whose right modules are direct sums of indecomposable modules, Proc. Amer. Math. Soc. 77 (1979), 191-197.

12. - Purity, algebraic compactness, direct sum decompositions, and representation type, in Proc. Euroconference on Infinite Length Modules (Bielefeld 1998) (to appear).

13. H. Hullinger, Stable equivalence and rings whose modules are a direct sum of finitely generated modules, J. Pure Appl. Algebra 16 (1980), 265-273.

14. G. Koethe, Verallgemeinerte abelsche Gruppen mit hyperkomplexem Operatorenring, Math. Zeitschr. 39 (1935), 31-44.

15. H. Lenzing and F. Okoh, The separability of direct products of modules over a canonical algebra, in Representations of Algebras (Ottawa 1992) (V. Dlab and H. Lenzing, Eds.), Canad. Math. Soc. Conf. Proc. Series 14 (1993), 339-352.

16. C.M. Ringel and H. Tachikawa, QF-3 rings, J. reine angew. Math. 272 (1975), 49-72.

17. D. Simson, A class of potential counterexamples to the pure semisimplicity conjecture, in Advances in Algebra and Model Theory (M. Droste and R. Göbel, eds.), Algebra Logic and Applications Series 9, Gordon and Breach, Amsterdam, 1997, pp. 345-373.

18. _ Dualities and pure semisimple rings, in Abelian Groups, Module Theory, and Topology (Padova 1997) (D. Dikranjan and L. Salce, eds.), Lecture Notes in Pure and Appl. Math., vol. 201, Dekker, New York, 1998, pp. 381-388.

19. R.B. Warfield, Jr., Purity and algebraic compactness for modules, Pac. J. Math. 28 (1969), 699-719.

20. W. Zimmermann, Rein-injektive direkte Summen von Moduln, Communic. in Algebra 5 (1977), 1083-1117.

21. _ Einige Charakterisierungen der Ringe ueber denen reine Untermoduln direkte Summanden sind, Bayer. Akad. Wiss. Math.-Natur. Kl. S.-B. 1972, Abt. II (1973), 77-79.

Department of Mathematics, University of California, Santa Barbara, CA 93106

E-mail address: birge@math.ucsb.edu

Department of Mathematics, Wayne State University, Detroit, Mi 48202

E-mail address: okoh@math.wayne.edu 